\title{
Research on the Ways to Improve the Overseas Communication of Chinese Symphony Against the Background of "the Belt and Road Initiative"
}

\begin{abstract}
Genghua Qiu, ${ }^{1, *}$
${ }^{1}$ College of Music, Central China Normal University, Wuhan, Hubei, China

*Corresponding author. Email: 122434172@qq.com

ABSTRACT

Since "the Belt and Road Initiative" was first put forward, people mostly observed and thought about it from the perspective of economic development and political cooperation. However, with the continuous deepening of "the Belt and Road Initiative" in recent years, cultural exchanges continue to integrate with international economic cooperation and political mutual trust. With economic and political cooperation, a large amount of cultural communication and exchange activities have existed between China and countries along the route. In this context, when cultural activities and the issue of building national cultural soft power come together, it is required to start from specific art categories and consider how to enhance cultural communication from the perspective of micro and macro. As an important art form, symphony is worthy of in-depth research and discussion on how to demonstrate its communication power and achieve communication effects in overseas communication against the Background of "the Belt and Road Initiative".
\end{abstract}

Keywords: "The Belt and Road Initiative", Chinese symphony, Overseas communication, Realistic path.

\section{INTRODUCTION}

With the integration of Chinese music elements in the process of local development, it has evolved from purely western "imports" to the Chinese symphony. Chinese symphony has developed from being simple to being perfect, and become the main force in the overseas communication of Chinese culture. And it has undergone more than a century of transformation from "localization" to "internationalization". "The communication is dissemination. People transfer information, ideas, attitudes and feelings through symbols to realize the information sharing and exchange, which is the socalled communication." [1] Taking Chinese symphony as the research object from the perspective of music communication, this paper analyzes the current situation of overseas communication of Chinese symphony, and discusses the differences and commonalities of overseas people's cognition of Chinese music

*Fund: Supported by the Fundamental Research Funds for the Central Universities (CCNU20A03002). culture, artistic expectation and interaction mode. At the same time, this paper explores the realistic path to enhance the overseas communication of Chinese symphony, and provides practical reference and inspiration for the creation of Chinese symphony works in the new era and the new strategy of overseas communication of Chinese music.

\section{PROSPECTS FOR OVERSEAS COMMUNICATION OF CHINESE SYMPHONY AGAINST THE BACKGROUND OF "THE BELT AND ROAD INITIATIVE"}

At the National Symphony Creation and Development Symposium hosted by the Chinese Musicians Association in January 2019, the industry participants placed great expectations on the development of symphony. Symphony, as the highest form of national music expression, has received more attention from the central government and government departments at all 
levels in recent years. At the same time, symphony can be regarded as the "heavy instrument" in music art for a country and is an important reference to measure a country's "soft power". [2]

The industry's expectation of the symphony is closely related to its unique position in the art of music. In the nearly 400 years of development, with the continuous accumulation of achievements by numerous artists in its long-term development process, symphony has formed its own theory and appreciation system. Meanwhile, symphony orchestra has outstanding performance and appeal compared with other musical art forms due to the richness of its organizational system of instruments. From the perspective of cross-cultural communication of music art, people can clearly see the far-reaching influence of symphonic works of Shostakovich, Tchaikovsky and other musicians in the Soviet Union on the development of music and art appreciation in China by reviewing the foreign symphonic works that have profound impact on China in history. Therefore, in order to build a three-dimensional communication system of Chinese culture abroad, symphony, as an important part of music art, should be an indispensable part.

Apart from the artistic characteristics and value of symphony itself, its communication is of great significance from the perspective of broadening international cultural exchanges and enhancing cultural communication ability. With the reform of communication media, the music communication media and methods are undergoing profound changes, and the effect of music communication has a greater space of activity in the "borderless and boundless" communication field. Compared with the communication of other contents, the music communication is easy to understand, easy to remember and extensible. The way of producing such communication effect is that "after receiving the musical information and producing psychological effect (understanding), the physical senses of the music recipient will transmit some feedback information to the communicator." [3] In this way, musicians can arouse the audience's emotional resonance and establish cultural identity in cross-cultural communication. With the advancement of "the Belt and Road Initiative", it is necessary to deepen mutual understanding through cultural exchanges with various arts as carriers in exchanges and cooperation with countries along the route, eliminate cultural barriers in international exchanges, and complete a higher level of economic and political cooperation on the basis of establishing a sense of cultural identity. Therefore, symphony, as an important carrier of art, has a brilliant future.

\section{THE STATUS QUO OF OVERSEAS COMMUNICATION OF CHINESE SYMPHONY}

Compared with western countries, which are the birthplaces of symphony, China's symphony started late with a weak foundation. With the rapid development of China's economy and society since the reform and opening up, the level of artistic and cultural undertakings has been rising rapidly, but the performance of symphony in overseas communication is still relatively inadequate, which is mainly reflected in the two aspects of the work itself and the communication carrier.

In the aspect of works, there is no denying the fact that since reform and opening up, a batch of well-known symphonic works have emerged, such as Chen Yi's orchestral music "Duo Ye", Chen Qigang's Cello Concerto "Reflet D'un Temps Disparu", Guo Wenjing's Flute Concerto "Chou Kong Shan", Qu Xiaosong's Symphonic Suite "Shan Yu Tu Feng", and Liu Yuan's orchestral music "The Echoes of Hakka's Earth Buildings". The creations of these composers are bold and novel, approaching and reaching the world level. However, many people in the music circle also point out that the creation of Chinese symphonic works is "neither foreign nor local". For example, Liu Jian believes that "the history of symphonic creation in the past 60 years is the history of Chinese composers struggling to 'establish' western orchestras" [4]. In his opinions, in addition to the technical difficulties and the influence of western music, Chinese symphonic composers still have a heavy burden of "searching for roots" and "making innovation". Compared with the perceptual tradition of traditional Chinese music, symphony, as a western import, has a three-dimensional sense of scene and requires logical levels, which is a completely different music system. In this context, faced with the "Chinese plot", the existing Chinese symphony works have not given a good answer.

At the same time, in terms of communication carriers, Chinese symphony presents the problems of single carrier type and insufficient number in overseas communication. For a long time, the overseas communication of Chinese symphony mainly relies on the communication of works and the orchestra's tour around the world. In terms of works, it is found that in 1988, the New York City Symphony Orchestra performed Tan Dun's "The 
Great Wall", "Peking Opera Adaptation" and other works in a concert at Avery Fisher Hall on Sunday. In recent years, He Zhanhao's and Chen Gang's "Liang Shanbo and Zhu Yingtai", Chen Qigang's "Five Elements" and other excellent works have become the repertoire of foreign orchestras. In terms of orchestra tour, with the continuous rise of China's international influence and the prosperity of symphony orchestra, the frequency of overseas tour has been increasing since the end of last century. The above two carriers have played an immeasurable role in promoting the development of orchestras and promoting Chinese symphonic music to connect with the world, and are also the best ways to enter the world symphony circle. However, in recent years, the transformation of music communication mode and the situation of COVID-19 prevention and control have dealt a heavy blow to the previous two modes of communication which are based on field performance. In foreign countries, with the application of symphony in games, film and television works, and online music performance relying on VR, HIFI and other technologies have become a new trend of symphony communication. How to grasp the trend and enrich the carrier to improve the communication power is a topic worthy of our consideration.

\section{REALISTIC PATHS TO IMPROVE THE OVERSEAS COMMUNICATION POWER OF CHINESE SYMPHONY}

Based on the current situation and the basic "5W" theory of communication, the overseas communication of Chinese symphony should focus on the communicator, content, audience and media. The overseas communication power can be improved by means of joint operation of contents about "Chinese style" at home and abroad, carrying out communication activities at a higher level to reach audiences and expanding the scope of communication, and using new media carriers to carry out symphony communication.

\subsection{Mobilizing the Overseas Human Resources to Select and Process Communication Contents Based on "Chinese Style"}

For a long time, people in the field of China's symphony persist in creating "Chinese style". As early as 1983, Qu Wei, the composer, pointed out in an article in "People's Music", "we must listen to the voice of the people, grasp the pulse of the times and create symphonic music with the spirit of the socialist of the times and Chinese style to meet the growing aesthetic requirements of the people." [5] In the practice of several generations of artists in China, this concept has gradually become concrete. The Chinese symphony has made great progress and formed its own characteristics.

Based on the disseminators and communication content, against the background of "the Belt and Road Initiative", the overseas communication of Chinese symphony should adhere to its own unique "Chinese style", but the maintenance of this characteristic requires a combination of management wisdom. And its communication content must be combined with the cultural characteristics and needs of the countries along the route. In this process, it is noted that a large number of Chinese musicians living abroad have excellent musical qualities and a good understanding of the audience and cultural characteristics of their host countries. It is better to listen to their opinions and mobilized their wisdom on how to re-create and disseminate their unique "Chinese style" abroad. "We should advocate the use of equality, mutual respect attitude to treat the international communication of Chinese national music culture. Neither can we measure Chinese culture with the standards of other countries or other nations, nor can we substitute the musical aesthetics of one country or one nation for the musical aesthetics of another country or another nation. We should respect each other's cultural views, musical views, aesthetic systems and values." [6]

Therefore, in the process of overseas communication of various Chinese symphony cultures in the future, it is required to play the role of overseas musicians, and make the planning together, especially around the selection and processing of communication contents, so as to maximize the communication effect. And then, symphony works and performances with "Chinese style" are promoted along the route.

\subsection{Improving the Level of Communication Activities and Combining the Characteristics of the Audience in the Organization and Arrangement}

The "Belt and Road Initiative" is an inclusive and open international regional cooperation system. 
The extensive cooperation between countries along the route and China in the field of economy and trade provides a good opportunity for various cultural exchange activities. At the same time, the development level of art and culture in the countries along the route is uneven. In the process of going out, we can learn the advanced experience of other countries and spread the Chinese symphony culture at the same time. In this context, it is required to seize opportunities and avoid the problems in the process of symphony communication, and improve the level of communication activities as much as possible, in order to have a greater impact and create more conditions for the exertion of communication power.

"Music does not express individual and certain happiness, sorrow, pain, fear, joy or peace of mind, but the happiness, sorrow, fear, joy and peace of mind itself, which is abstract to a certain extent." [7] Considering the overseas audience of Chinese symphony, musician used to perform some official artistic performances, ignoring the audiences' acceptance and aesthetic needs. This subjective propaganda model not only has a strong political color, but also ignores the audience's choice of music, affecting the communication effect of symphony performances and exchange activities. In the future, in the process of overseas communication of symphony in the countries along the route, it is required to pay attention to specific problems and specific analysis, pay special attention to understanding the needs of the audiences, and create a new communication mode that suits cultural background, way of thinking and appreciation habits of the audiences, so as to truly achieve a two-way communication in a state of "dialogue".

\subsection{Focusing on Social and Technological Changes and Exploring New Methods of Organizational Operation and Communication Carriers}

With the changes of modern social life, on the one hand, the audience of symphonic performance is constantly expanding, on the other hand, its interpretation methods and carriers are also constantly expanding. In order to enhance the overseas communication against the background of "the Belt and Road Initiative", it is better to closely follow up the development trend and promote Chinese symphony to more audiences in countries along the route through three-dimensional distribution of cultural products and promotion of new technologies.

In terms of organization and operation, it is necessary to encourage more exchanges of folk music and open up new organizational models. Starting from the cultural attributes of music communication, it has its own characteristics compared with economic and trade exchanges and political cooperation. Therefore, compared with the "government-run exchanges" that are predominant in the past, it is necessary to encourage more nongovernmental organizations to join in the future, so that they can cooperate with government departments in various forms. In the overseas communication of Chinese symphony, resources such as capital and talents and communication channels should be more fully mobilized to form an organization and operation pattern of multi-party cooperation and official and non-governmental cooperation.

In addition, new communication carriers should be opened up in light of the current technology and industrial development situation. What is particularly prominent at the moment is the cooperation with other cultural products such as movies and games, as well as the dissemination of new media technologies. With the development of the cultural industry, from arrangement to harmony, orchestration to recording, as well as postproduction and distribution, the procedures of modern symphony are basically consistent with the processes of film distribution, game production, etc., with typical industrial production characteristics. At the same time, cultural products such as movies and games also have a huge demand for music. For example, the game "World of Warcraft" has achieved the joint success of music creation and game creation through the creation and adaptation of systematic symphony works, being in line with the different styles of environment, region, ethnicity and other backgrounds. The background of famous movie "Pirates of the Caribbean" has become a guest of many worldrenowned orchestras in the form of a symphonic suite. These precedents are worthwhile for us to find a new model for symphony communication in the process of Chinese cultural products going to the countries along the "Belt and Road" route, so as to achieve a win-win situation of economic benefits and cultural communication benefits. 


\section{CONCLUSION}

To sum up, in the context of "the Belt and Road Initiative", promoting the Chinese symphony to move toward countries along the route is a comprehensive and long-term topic for enhancing the country's cultural dissemination strength and promoting economic, trade and political exchanges with countries along the route. In this process, in order to enhance the dissemination of Chinese symphony, on the one hand, it is required to combine the artistic characteristics of symphony, on the other hand, it is better to respect the law of communication, and carry out activities in accordance with the actual conditions of music, art and cultural communication of countries along the route. Starting from this, it is required to improve the work level of communicators, study the characteristics of the audience, explore new carriers, organize communication activities scientifically, and allocate communication resources, so as to provide practical support for the promotion of communication effects and the construction of the communication system.

\section{AUTHORS' CONTRIBUTIONS}

This paper is independently completed by Genghua Qiu.

\section{REFERENCES}

[1] Ci Hai. The 1999 compact edition, the first edition in January 2002, the 13th edition in April 2008, 232.

[2] Pei Nuo. Consolidating the consensus of China's symphony undertaking in the new era - The National Symphony Creation and Development Symposium in Beijing $[\mathrm{J}]$. Music creation, 2019, (3). (in Chinese)

[3] Yang Dan. School music education from the sociological perspective $[\mathrm{J}]$. Journal of Hubei University for Nationalities (Philosophy and Social Sciences), 2012, 30(06): 126-130+135. (in Chinese)

[4] Liu Jian. "Well-leveraged": Listening to "Chinese Overture - Chinese Symphonic Works" [J]. People's Music, 2018(01): 22-24. (in Chinese)

[5] Qu Wei. Creation of symphonic music works with Chinese style [J]. People's Music, 1983 (06): 5-9. (in Chinese)
[6] Wang Yaohua. The International Communication and Promotion of Chinese National Music Culture - The level, adaptability, "change" and "invariability" of the communication content [J]. Journal of the Central Conservatory of Music, 2014 (03), 95100. (in Chinese)

[7] Kremlyov. Introduction to Music Aesthetics [M]. Music Publishing House, 1959, 107. (in Chinese) 